\title{
Ground Glass Nuclear Inclusion
}

National Cancer Institute

\section{Source}

National Cancer Institute. Ground Glass Nuclear Inclusion. NCI Thesaurus. Code C36018.

A microscopic finding indicating the presence of nuclei with ground glass appearance due to the presence of intranuclear viral particles that cause peripheral marg ination of the chromatin. 